\title{
The Economic Outcomes of Pharmacist Interventions in Total Parenteral Nutrition Services in Saudi Arabia
}

\author{
Yousef Ahmed Alomi* iD, The \\ Former General Manager of General \\ Administration of Pharmaceutical Care. \\ Former Head, National Clinical Pharmacy \\ and Pharmacy Practice, Former Head, \\ Pharmacy R\&D Administration, Ministry of \\ Health, Riyadh, SAUDI ARABIA. \\ Aisha Omar Fallatah, Head, IV \\ Admixture and TPN Services, King Salman \\ Hospital, Ministry of Health, Riyadh, SAUDI \\ ARABIA. \\ Faiz A. Bahadig, Informatics Pharma- \\ cist, Pharmaceutical Care Department, \\ King Abdul-Aziz Medical, City-WR-Jeddah, \\ Ministry of National Guard, SAUDI ARABIA. \\ Amjad Ahmad AL Qahtani, Clinical \\ Pharmacy Staff, General Administration of \\ Pharmaceutical Care, Ministry of Health, \\ Riyadh, SAUDI ARABIA.
}

\section{Correspondence:}

Dr. Yousef Ahmed Alomi, TThe Former General Manager of General Administration of Pharmaceutical Care, Former Head, National Clinical Pharmacy and Pharmacy Practice, Former Head, Pharmacy R\&D Administration, Ministry of Health, Riyadh-11392, SAUDI ARABIA.

Phone no: +966504417712

E-mail:yalomi@gmail.com
Received: 13-12-2018

Approved: 10-2-2019

Copyright: (c) the author(s),publisher and licensee Pharmacology, Toxicology and Biomedical Reports. This is an open-access article distributed under the terms of the Creative Commons Attribution NonCommercial License, which permits unrestricted non-commercial use, distribution, and reproduction in any medium, provided the original work is properly cited.

This is an open access article distributed under the terms of the Creative Commons Attribution-NonCommercial-ShareAlike 4.0 License

Access this article online

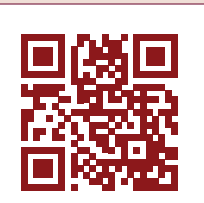

www.ptbreports.org

DOI:

10.5530/PTB.2019.5.36

\begin{abstract}
Objectives: There are several problems and complications related to the Total Parenteral Nutrition (TPN) services. The pharmacist plays a crucial role in preventing these problems. Therefore, in this study, we aimed to assess the economic outcomes of pharmacist intervention in cost avoidance related to TPN services at a public hospital in Riyadh city, Saudi Arabia. Methods: Cohort prospective analysis of data collected regarding pharmacist intervention in TPN services provided to neonates, pediatrics and adult patients was performed. TPN services were provided $8 \mathrm{hr}$ in a day and 7 days in a week. The study site was a 300-bed public hospital at the Ministry of Health (MOH) in Riyadh city, Saudi Arabia. The pharmacist reviews and prepares TPN and he pharmacist documents any or all TPN-related problems before the preparation of TPN or after dispensing it. The pharmacist uses an International Study Model to measure level of activity and provides rational of clinical intervention, recommendation, patient outcome, impact of pharmacoeconomics and related cost analysis. Results: The pharmacist identified a total of 402 TPN-related problems. The total number of TPN services provided was 394 prescribed to 82 patients. Of these, $209(51.99 \%)$ and $193(48.01 \%)$ interventions were provided to males and females, respectively. The total cost avoidance from pharmacist intervention in 1 year was 578,926.89 USD. The highest cost avoidance of TPN interventions was recorded for potentially significant $154.90 \%$; $100,040.91$ USD) and potentially serious problems (33.33\%; 244,696.83 USD). The maximum cost avoidance in the case of rational clinical activities was recorded for TPN consultations (325,695.76 USD) followed by incompatibility $(275,802.66)$ and inappropriate route $(189,912.34$ USD). The maximum cost avoidance related to patient outcome was recorded for patient condition improved (489,830.93 USD) and laboratory value improved $(89,095.96)$. The maximum pharmacoeconomic cost avoidance impact was the patient duration of hospital stay decreased $(226 ; 332,220.70$ USD) and reduction in the drug therapy monitoring $(246,227.15$ USD). Conclusion: The clinical pharmacist responsible for TPN services plays an essential role in preventing TPN-related problems and avoid the unnecessary economic burden on the healthcare system. Expanding the role TPN clinical pharmacist is highly recommended for all TPN services to reduce the economic burden on healthcare system in Saudi Arabia.

Key words: Economic outcome, Pharmacist intervention, Total parenteral nutrition, Ministry of Health, Saudi Arabia.
\end{abstract}

\section{INTRODUCTION}

The strategic plan on pharmacy was released in 2012 with several general goals and initiatives. ${ }^{1}$ The new and updated version of this plan along with the current evaluation and inclusion of New Saudi Vision 2030 was released in $2018 .^{2}$ This version states several tools for monitoring the plan, implementation and outcomes of the pharmacy practice programs. ${ }^{1}$ The cost avoidance or cost impact was one of the current application tools. ${ }^{3}$ Several international publications have explored the impact of cost avoidance on the pharmacist performance in different clinical pharmacy programs including nutrition support pharmacy. ${ }^{4-6}$ In addition, several local investigations used the tool and showed the high-cost impact of some pharmacy programs. ${ }^{7-10}$ However, to the best of our knowledge, there are no studies conducted on the nutrition support services and their economic impact and cost avoidance in the KSA, Gulf and Middle Eastern countries. Therefore, in this study, we aimed to explore the economic impact of pharmacist interventions with nutrition support services at a public hospital located in Riyadh city, KSA.

\section{METHODS}

This is a cohort prospective analysis of 12-month data of 2015 regarding pharmacist intervention by providing TPN services to neonates, pediatrics and adult patients. TPN services are provided at the public hospital belonging to the $\mathrm{MOH}$; it provides services for $8 \mathrm{hr}$ in a day and 7 days in a week and has 300 beds. The hospital has following specialties: adult's endocrinology, adult's nephrology pediatrics, adult's cardiology, adults internal medicine, adults surgery, adults critical care, NICU, PICU and obstetrics and gynecology. The hospital has ambulatory care services and emergency services with above specialties. In addition, the hospital had comprehensive pharmaceutical services including inpatient pharmacy and ambulatory care pharmacy. Furthermore, the TPN services, drug information services and computerized physician order entry system is available at both inpatient and outpatient units. In 2009s, the pharmacy services established TPN services starting from physician prescribing it to pharmacist reviewing, preparing and dispensing it; the nurse administers the preparation to the patients. After 5 years, the pharmacy improved the services by in- 
stalling the automated compounding system techniques. TPNrelated problems were documented before preparation and after dispensing the TPN order. The pharmacist intervention used an International Study Model, which measures the level of activity and provides rational of clinical intervention, recommendation, patient outcome impact of pharmacoeconomics and related cost analysis. ${ }^{11,12}$ The data were gathered and analyzed by using the Survey Monkey system.

\section{RESULTS}

The pharmacist identified a total of 402 TPN-related problems. A total of 394 TPN orders were prescribed to 82 patients. The rate of intervention was 4.9 per patient. Of those, 209 (51.99\%) and 193 (48.01\%) were provided to males and females, respectively. The majority of patients were Saudi (266 (66.2\%)) patients (non-Saudi patients were $136(33.8 \%)$ in number). The majority of patients were neonates (303 (75.56\%)) followed by pediatrics $(97(24.19 \%))$ ( 1$)$. The total cost avoidance from pharmacist intervention for 1 year was 578,926.89 USD. The maximum cost avoidance in female patients was $47.06 \%$ (299,630.67 USD), whereas the maximum cost avoidance in males was $52.94 \%$ (279,296.22 USD). The maximum nationality-related cost avoidance was 59.61\% (354,036.65 USD) in Saudi patients, whereas it was $40.39 \%$ (224,890.24 USD) in non-Saudi patients. The maximum cost avoidance in neonates was $76.47 \%(403,762.96$ USD) and in pediatrics, it was $23.53 \%(175,163.93$ USD) (Tables 2-4). The maximum cost avoidance was recorded for senior registrar prescriber $(67.84 \% ; 319,488.32$ USD) and consultant prescriber $(27.06 \% ; 209,323.17$ USD). The maximum cost avoidance in terms of TPN interventions was recorded for potentially significant (54.90\%; 100,040.91 USD) and potentially serious (33.33\%; 244,696.83 USD) problems (Tables 5 and 6). The maximum rational cost avoidance in terms of clinical activities was recorded for TPN consultations
Table 1: Patient demographic information.

\begin{tabular}{|c|c|c|}
\hline \multicolumn{3}{|l|}{ Age } \\
\hline Answer Options & Response Count & Response Percent \\
\hline 0-30 days & 303 & $75.6 \%$ \\
\hline 1 month- 6 years & 97 & $24.2 \%$ \\
\hline $6-12$ years & 1 & $0.2 \%$ \\
\hline $12-18$ years & 0 & $0.0 \%$ \\
\hline $18-40$ years & 0 & $0.0 \%$ \\
\hline $40-65$ years & 0 & $0.0 \%$ \\
\hline 65 or older & 0 & $0.0 \%$ \\
\hline \multicolumn{2}{|l|}{ answered question } & 401 \\
\hline \multicolumn{2}{|l|}{ skipped question } & 1 \\
\hline \multicolumn{3}{|l|}{ Sex } \\
\hline Answer Options & Response Count & Response Percent \\
\hline Female & 193 & $48.0 \%$ \\
\hline Male & 209 & $52.0 \%$ \\
\hline \multicolumn{2}{|l|}{ answered question } & 402 \\
\hline \multicolumn{2}{|l|}{ skipped question } & 0 \\
\hline \multicolumn{3}{|l|}{ Nationality } \\
\hline Answer Options & Response Count & Response Percent \\
\hline Saudi & 266 & $66.2 \%$ \\
\hline Non-Saudi & 136 & $33.8 \%$ \\
\hline \multicolumn{2}{|l|}{ answered question } & 402 \\
\hline \multicolumn{2}{|l|}{ skipped question } & 0 \\
\hline
\end{tabular}

Table 2: Gender distribution versus cost avoidance.

\begin{tabular}{|c|c|c|c|c|c|c|c|c|c|c|}
\hline \multirow{2}{*}{$\begin{array}{l}\text { Sex } \\
\text { No }\end{array}$} & \multirow[b]{2}{*}{ Cost } & \multicolumn{3}{|c|}{ Female } & \multicolumn{3}{|c|}{ Male } & \multicolumn{3}{|c|}{ Total } \\
\hline & & No & $\%$ & Cost avoidance & No & $\%$ & Cost avoidance & No & $\%$ & Cost avoidance \\
\hline 1a & 26.17 & 0 & $0.00 \%$ & 0.00 & 0 & $0.00 \%$ & 0.00 & 0 & $0.00 \%$ & 0.00 \\
\hline $1 b$ & 54.89 & 0 & $0.00 \%$ & 0.00 & 0 & $0.00 \%$ & 0.00 & 0 & $0.00 \%$ & 0.00 \\
\hline $2 \mathrm{a}$ & 30.35 & 0 & $0.00 \%$ & 0.00 & 0 & $0.00 \%$ & 0.00 & 0 & $0.00 \%$ & 0.00 \\
\hline $2 b$ & 30.35 & 0 & $0.00 \%$ & 0.00 & 0 & $0.00 \%$ & 0.00 & 0 & $0.00 \%$ & 0.00 \\
\hline $2 c$ & 30.35 & 0 & $0.00 \%$ & 0.00 & 0 & $0.00 \%$ & 0.00 & 0 & $0.00 \%$ & 0.00 \\
\hline $2 d$ & 30.35 & 3 & $60.00 \%$ & 91.05 & 2 & $40.00 \%$ & 60.70 & 5 & $1.96 \%$ & 151.75 \\
\hline $2 \mathrm{e}$ & 30.35 & 0 & $0.00 \%$ & 0.00 & 0 & $0.00 \%$ & 0.00 & 0 & $0.00 \%$ & 0.00 \\
\hline $2 \mathrm{f}$ & 34.31 & 0 & $0.00 \%$ & 0.00 & 0 & $0.00 \%$ & 0.00 & 0 & $0.00 \%$ & 0.00 \\
\hline $3 a$ & 260.46 & 6 & $46.15 \%$ & $1,562.76$ & 7 & $53.85 \%$ & $1,823.22$ & 13 & $5.10 \%$ & $3,385.98$ \\
\hline $3 b$ & 109.00 & 8 & $66.67 \%$ & 872.00 & 4 & $33.33 \%$ & 436.00 & 12 & $4.71 \%$ & $1,308.00$ \\
\hline $3 c$ & 320.70 & 1 & $14.29 \%$ & 320.70 & 6 & $85.71 \%$ & $1,924.20$ & 7 & $2.75 \%$ & $2,244.90$ \\
\hline $4 \mathrm{a}$ & 370.04 & 62 & $44.29 \%$ & $22,942.48$ & 78 & $55.71 \%$ & $28,863.12$ & 140 & $54.90 \%$ & $51,805.60$ \\
\hline $4 \mathrm{~b}$ & 581.75 & 1 & $25.00 \%$ & 581.75 & 3 & $75.00 \%$ & $1,745.25$ & 4 & $1.57 \%$ & $2,327.00$ \\
\hline $4 c$ & $3,562.79$ & 9 & $60.00 \%$ & $32,065.11$ & 6 & $40.00 \%$ & $21,376.74$ & 15 & $5.88 \%$ & $53,441.85$ \\
\hline $4 \mathrm{~d}$ & $6,437.13$ & 10 & $58.82 \%$ & $64,371.30$ & 7 & $41.18 \%$ & $45,059.91$ & 17 & $6.67 \%$ & $109,431.21$ \\
\hline $5 a$ & $6,592.03$ & 6 & $31.58 \%$ & $39,552.18$ & 13 & $68.42 \%$ & $85,696.39$ & 19 & $7.45 \%$ & $125,248.57$ \\
\hline $5 b$ & $9,466.36$ & 12 & $66.67 \%$ & $113,596.32$ & 6 & $33.33 \%$ & $56,798.16$ & 18 & $7.06 \%$ & $170,394.48$ \\
\hline $5 c$ & $11,837.51$ & 2 & $40.00 \%$ & $23,675.02$ & 3 & $60.00 \%$ & $35,512.53$ & 5 & $1.96 \%$ & $59,187.55$ \\
\hline 6 & $100,000.00$ & 0 & $0.00 \%$ & 0.00 & 0 & $0.00 \%$ & 0.00 & 0 & $0.00 \%$ & 0.00 \\
\hline Total & & 120 & $47.06 \%$ & $299,630.67$ & 135 & $52.94 \%$ & $279,296.22$ & 255 & $100.00 \%$ & $578,926.89$ \\
\hline
\end{tabular}


Table 3: Nationality versus cost avoidance.

\begin{tabular}{|c|c|c|c|c|c|c|c|c|c|c|}
\hline \multirow{2}{*}{$\begin{array}{l}\text { Sex } \\
\text { No }\end{array}$} & \multirow[b]{2}{*}{ Cost } & \multicolumn{3}{|c|}{ Saudi } & \multicolumn{3}{|c|}{ Non-Saudi } & \multicolumn{3}{|c|}{ Total } \\
\hline & & No & $\%$ & Costavoidance & No & $\%$ & Cost avoidance & No & $\%$ & Cost avoidance \\
\hline $1 \mathrm{a}$ & 26.17 & 0 & $0.00 \%$ & 0.00 & 0 & $0.00 \%$ & 0.00 & 0 & $0.00 \%$ & 0.00 \\
\hline $1 b$ & 54.89 & 0 & $0.00 \%$ & 0.00 & 0 & $0.00 \%$ & 0.00 & 0 & $0.00 \%$ & 0.00 \\
\hline $2 a$ & 30.35 & 0 & $0.00 \%$ & 0.00 & 0 & $0.00 \%$ & 0.00 & 0 & $0.00 \%$ & 0.00 \\
\hline $2 \mathrm{~b}$ & 30.35 & 0 & $0.00 \%$ & 0.00 & 0 & $0.00 \%$ & 0.00 & 0 & $0.00 \%$ & 0.00 \\
\hline $2 c$ & 30.35 & 0 & $0.00 \%$ & 0.00 & 0 & $0.00 \%$ & 0.00 & 0 & $0.00 \%$ & 0.00 \\
\hline $2 \mathrm{~d}$ & 30.35 & 5 & $100.00 \%$ & 151.75 & 0 & $0.00 \%$ & 0.00 & 5 & $1.96 \%$ & 151.75 \\
\hline $2 \mathrm{e}$ & 30.35 & 0 & $0.00 \%$ & 0.00 & 0 & $0.00 \%$ & 0.00 & 0 & $0.00 \%$ & 0.00 \\
\hline $2 f$ & 34.31 & 0 & $0.00 \%$ & 0.00 & 0 & $0.00 \%$ & 0.00 & 0 & $0.00 \%$ & 0.00 \\
\hline $3 a$ & 260.46 & 7 & $53.85 \%$ & $1,823.22$ & 6 & $46.15 \%$ & $1,562.76$ & 13 & $5.10 \%$ & $3,385.98$ \\
\hline $3 b$ & 109 & 7 & $58.33 \%$ & 763.00 & 5 & $41.67 \%$ & 545.00 & 12 & $4.71 \%$ & $1,308.00$ \\
\hline $3 c$ & 320.7 & 3 & $42.86 \%$ & 962.10 & 4 & $57.14 \%$ & $1,282.80$ & 7 & $2.75 \%$ & $2,244.90$ \\
\hline $4 \mathrm{a}$ & 370.04 & 79 & $56.43 \%$ & $29,233.16$ & 61 & $43.57 \%$ & $22,572.44$ & 140 & $54.90 \%$ & $51,805.60$ \\
\hline $4 \mathrm{~b}$ & 581.75 & 4 & $100.00 \%$ & $2,327.00$ & 0 & $0.00 \%$ & 0.00 & 4 & $1.57 \%$ & $2,327.00$ \\
\hline $4 c$ & $3,562.79$ & 12 & $80.00 \%$ & $42,753.48$ & 3 & $20.00 \%$ & $10,688.37$ & 15 & $5.88 \%$ & $53,441.85$ \\
\hline $4 d$ & $6,437.13$ & 10 & $58.82 \%$ & $64,371.30$ & 7 & $41.18 \%$ & $45,059.91$ & 17 & $6.67 \%$ & $109,431.21$ \\
\hline $5 a$ & $6,592.03$ & 12 & $63.16 \%$ & $79,104.36$ & 7 & $36.84 \%$ & $46,144.21$ & 19 & $7.45 \%$ & $125,248.57$ \\
\hline $5 b$ & $9,466.36$ & 9 & $50.00 \%$ & $85,197.24$ & 9 & $50.00 \%$ & $85,197.24$ & 18 & $7.06 \%$ & $170,394.48$ \\
\hline $5 c$ & $11,837.51$ & 4 & $80.00 \%$ & $47,350.04$ & 1 & $20.00 \%$ & $11,837.51$ & 5 & $1.96 \%$ & $59,187.55$ \\
\hline 6 & $100,000.00$ & 0 & $0.00 \%$ & 0.00 & 0 & $0.00 \%$ & 0.00 & 0 & $0.00 \%$ & 0.00 \\
\hline Total & & 152 & $59.61 \%$ & $354,036.65$ & 103 & $40.39 \%$ & $224,890.24$ & 255 & $100.00 \%$ & $578,926.89$ \\
\hline
\end{tabular}

Table 4: Age distribution versus cost avoidance.

\begin{tabular}{|c|c|c|c|c|c|c|c|c|c|c|}
\hline Sex & & & $0-30 d$ & & & $1 \mathrm{mont}$ & years & & & \\
\hline No & Cost & No & $\%$ & Cost avoidance & No & $\%$ & Cost avoidance & No & $\%$ & Cost avoidance \\
\hline 1a & 26.17 & 0 & $0.00 \%$ & 0.00 & 0 & $0.00 \%$ & 0.00 & 0 & $0.00 \%$ & 0.00 \\
\hline $1 \mathrm{~b}$ & 54.89 & 0 & $0.00 \%$ & 0.00 & 0 & $0.00 \%$ & 0.00 & 0 & $0.00 \%$ & 0.00 \\
\hline $2 \mathrm{a}$ & 30.35 & 0 & $0.00 \%$ & 0.00 & 0 & $0.00 \%$ & 0.00 & 0 & $0.00 \%$ & 0.00 \\
\hline $2 b$ & 30.35 & 0 & $0.00 \%$ & 0.00 & 0 & $0.00 \%$ & 0.00 & 0 & $0.00 \%$ & 0.00 \\
\hline $2 c$ & 30.35 & 0 & $0.00 \%$ & 0.00 & 0 & $0.00 \%$ & 0.00 & 0 & $0.00 \%$ & 0.00 \\
\hline $2 \mathrm{~d}$ & 30.35 & 4 & $80.00 \%$ & 121.40 & 1 & $20.00 \%$ & 30.35 & 5 & $1.96 \%$ & 151.75 \\
\hline $2 \mathrm{e}$ & 30.35 & 0 & $0.00 \%$ & 0.00 & 0 & $0.00 \%$ & 0.00 & 0 & $0.00 \%$ & 0.00 \\
\hline $2 \mathrm{f}$ & 34.31 & 0 & $0.00 \%$ & 0.00 & 0 & $0.00 \%$ & 0.00 & 0 & $0.00 \%$ & 0.00 \\
\hline $3 a$ & 260.46 & 9 & $69.23 \%$ & $2,344.14$ & 4 & $30.77 \%$ & $1,041.84$ & 13 & $5.10 \%$ & $3,385.98$ \\
\hline $3 \mathrm{~b}$ & 109 & 11 & $91.67 \%$ & $1,199.00$ & 1 & $8.33 \%$ & 109.00 & 12 & $4.71 \%$ & $1,308.00$ \\
\hline $3 c$ & 320.7 & 4 & $57.14 \%$ & $1,282.80$ & 3 & $42.86 \%$ & 962.10 & 7 & $2.75 \%$ & $2,244.90$ \\
\hline $4 \mathrm{a}$ & 370.04 & 111 & $79.29 \%$ & $41,074.44$ & 29 & $20.71 \%$ & $10,731.16$ & 140 & $54.90 \%$ & $51,805.60$ \\
\hline $4 \mathrm{~b}$ & 581.75 & 3 & $75.00 \%$ & $1,745.25$ & 1 & $25.00 \%$ & 581.75 & 4 & $1.57 \%$ & $2,327.00$ \\
\hline $4 c$ & $3,562.79$ & 12 & $80.00 \%$ & $42,753.48$ & 3 & $20.00 \%$ & $10,688.37$ & 15 & $5.88 \%$ & $53,441.85$ \\
\hline $4 \mathrm{~d}$ & $6,437.13$ & 13 & $76.47 \%$ & $83,682.69$ & 4 & $23.53 \%$ & $25,748.52$ & 17 & $6.67 \%$ & $109,431.21$ \\
\hline $5 a$ & $6,592.03$ & 14 & $73.68 \%$ & $92,288.42$ & 5 & $26.32 \%$ & $32,960.15$ & 19 & $7.45 \%$ & $125,248.57$ \\
\hline $5 b$ & $9,466.36$ & 12 & $66.67 \%$ & $113,596.32$ & 6 & $33.33 \%$ & $56,798.16$ & 18 & $7.06 \%$ & $170,394.48$ \\
\hline $5 c$ & $11,837.51$ & 2 & $40.00 \%$ & $23,675.02$ & 3 & $60.00 \%$ & $35,512.53$ & 5 & $1.96 \%$ & $59,187.55$ \\
\hline 6 & $100,000.00$ & 0 & $0.00 \%$ & 0.00 & 0 & $0.00 \%$ & 0.00 & 0 & $0.00 \%$ & 0.00 \\
\hline Total & & 195 & $76.47 \%$ & $403,762.96$ & 60 & $23.53 \%$ & $175,163.93$ & 255 & $100.00 \%$ & $578,926.89$ \\
\hline
\end{tabular}




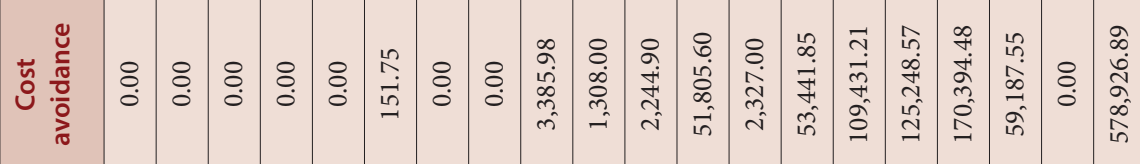

䒿 官

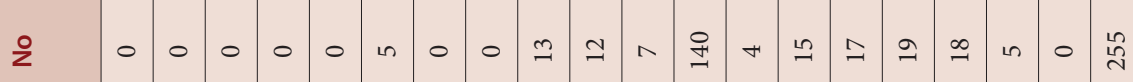

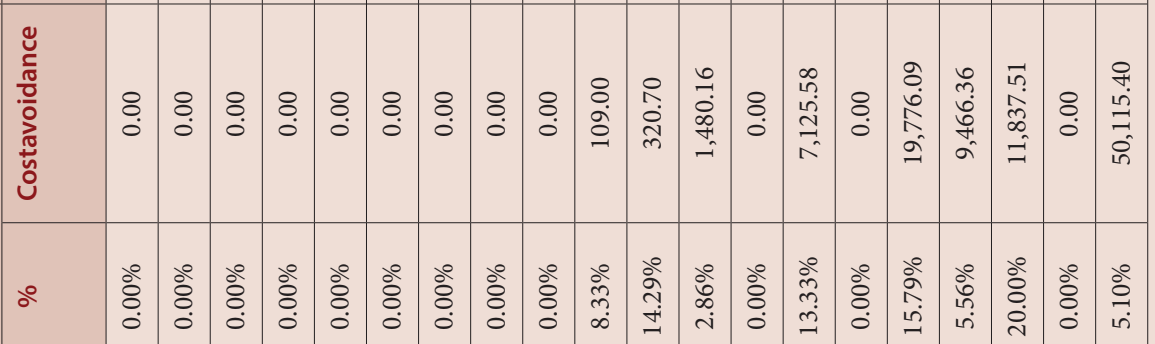

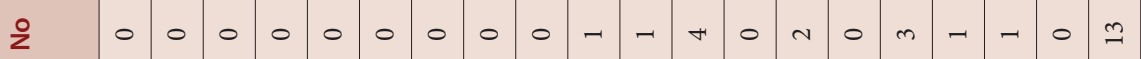

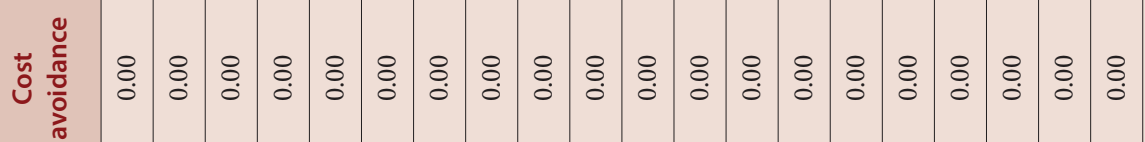

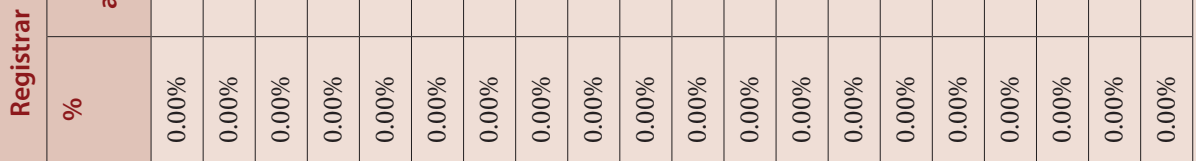
\begin{tabular}{ll|l|l|l|l|l|l|l|l|l|l|l|l|l|l|l|l|l|l|l} 
z & 0 & 0 & 0 & 0 & 0 & 0 & 0 & 0 & 0 & 0 & 0 & 0 & 0 & 0 & 0 & 0 & 0 & 0 & 0 & 0
\end{tabular}

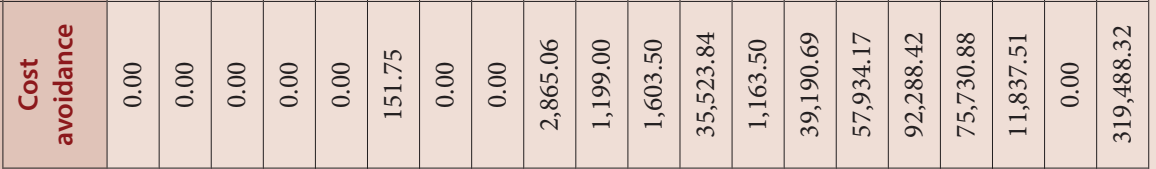

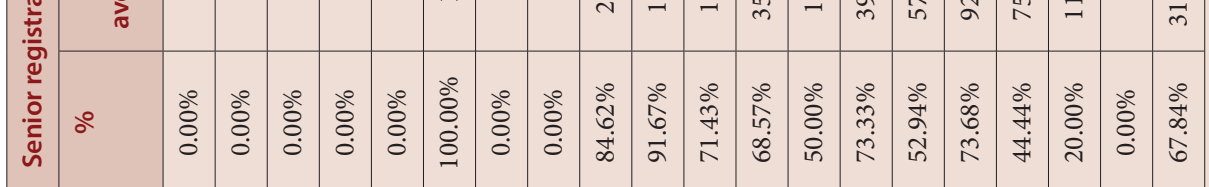

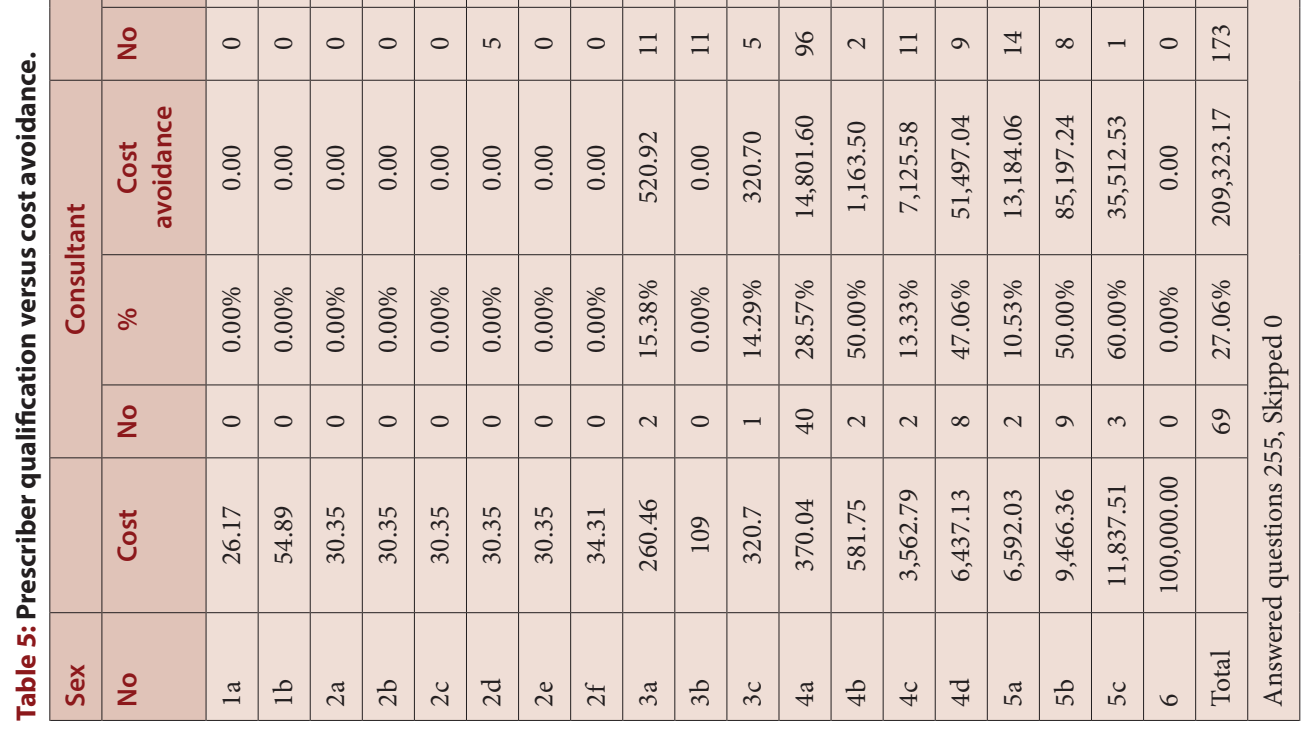




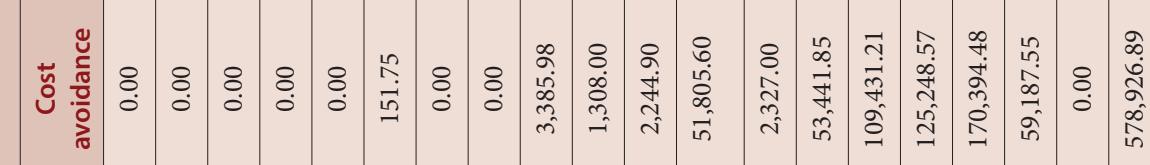

กัँ

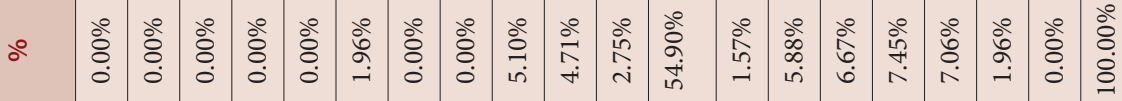

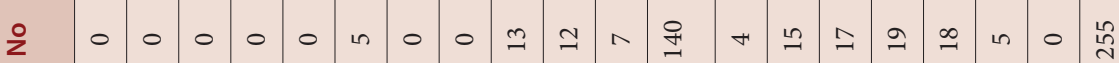

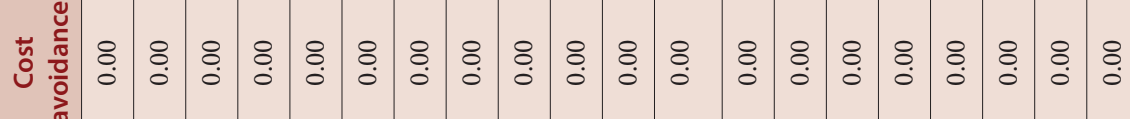

丞

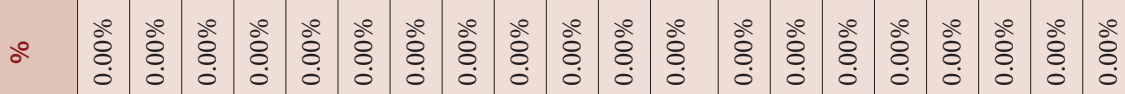

$\begin{array}{llllllllllllllllllllll} & 0 & 0 & 0 & 0 & 0 & 0 & 0 & 0 & 0 & 0 & 0 & 0 & 0 & 0 & 0 & 0 & 0 & 0 & 0 & 0 & 0\end{array}$

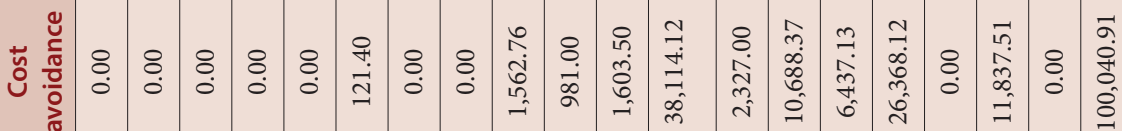

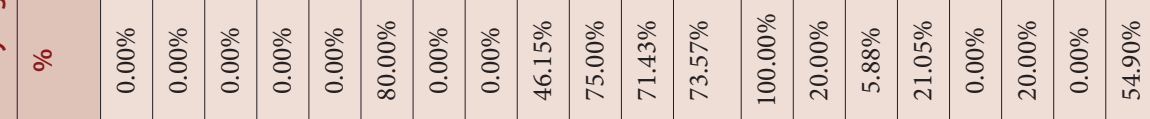

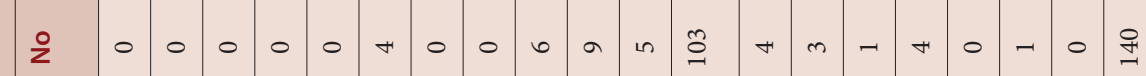

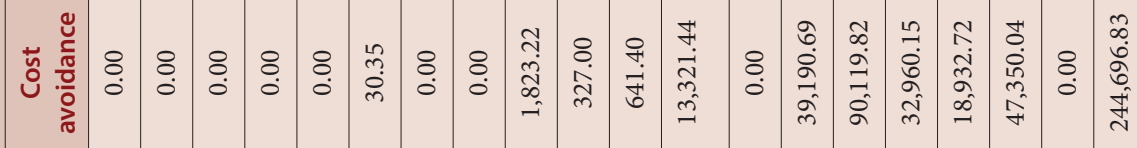

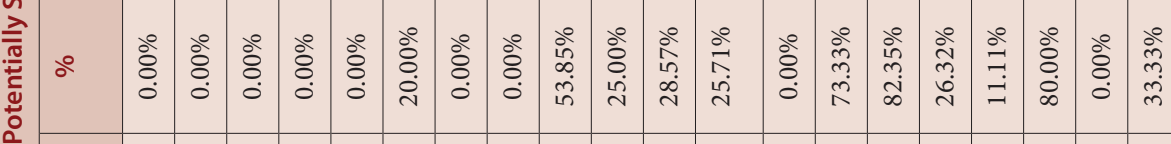

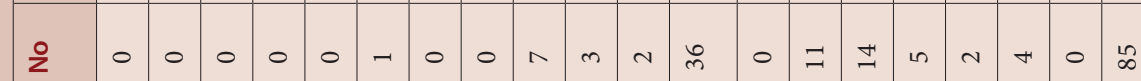

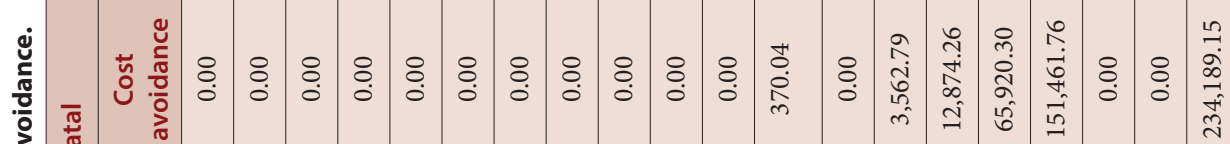

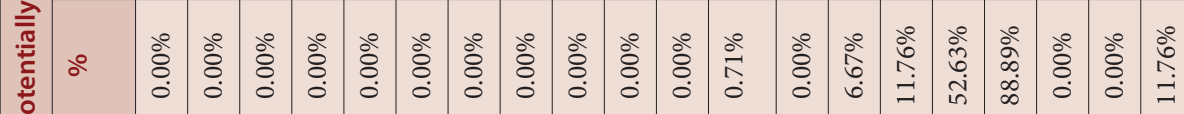

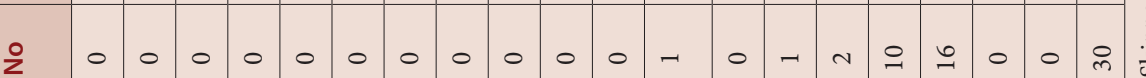

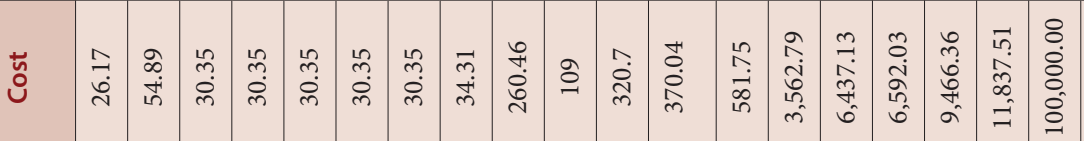

爻 


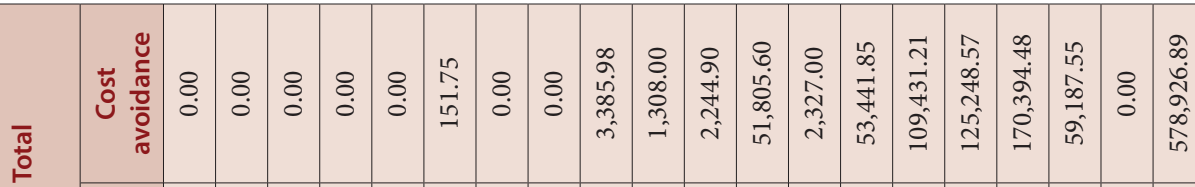

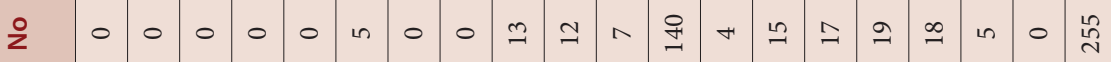

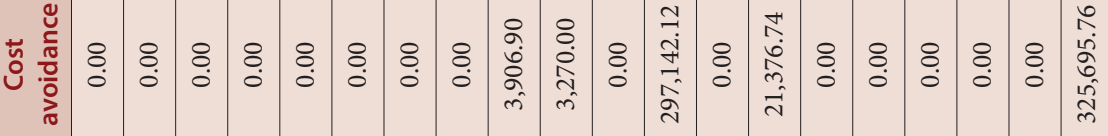

$\begin{array}{lllllllllllllllllllllllllllll}z & 0 & 0 & 0 & 0 & 0 & 0 & 0 & 0 & m & 0 & 0 & = & n & n & 0 & - & 0 & 0 & 0 & \bar{m}\end{array}$

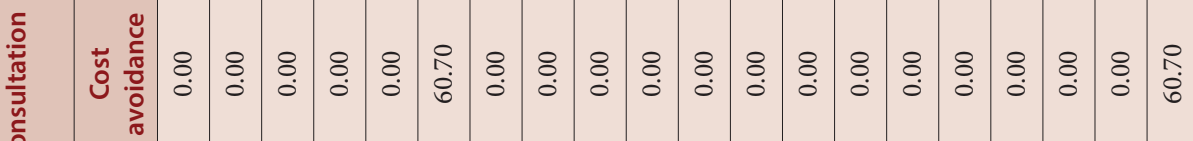

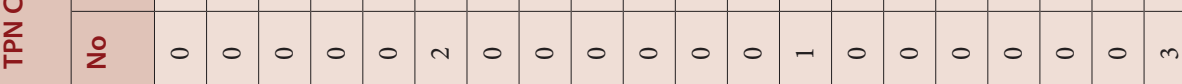

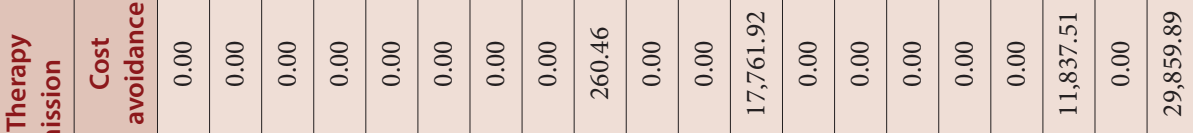

ํํㄹ

$\begin{array}{lllllllllllllllllllllllll}2 & 0 & 0 & 0 & 0 & 0 & 0 & 0 & 0 & -1 & 0 & 0 & \stackrel{\infty}{4} & 0 & 0 & 0 & 0 & 0 & -1 & 0 & \text { in }\end{array}$

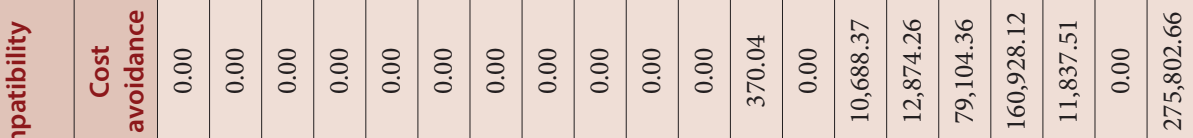

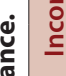

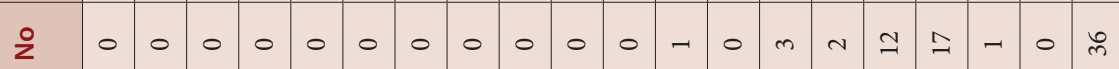

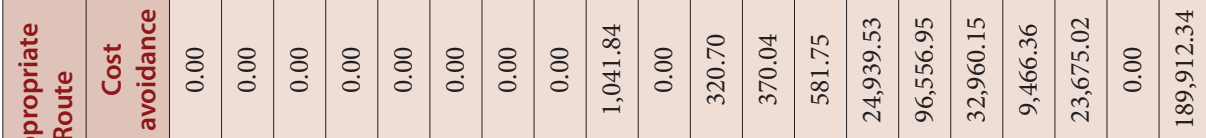

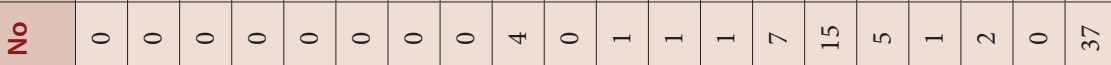

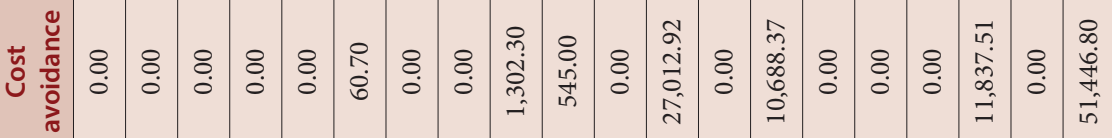

$\begin{array}{lllllllllllllllllllllllll}z & 0 & 0 & 0 & 0 & 0 & n & 0 & 0 & \ln & \ln & 0 & n & 0 & m & 0 & 0 & 0 & -1 & 0 & \infty\end{array}$

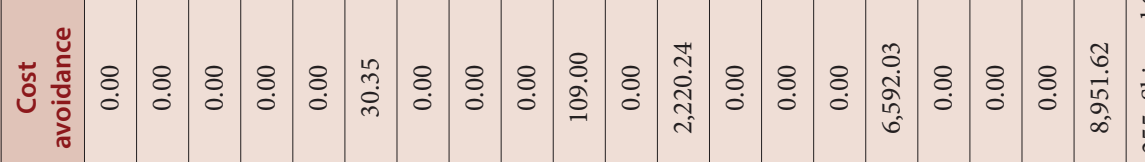

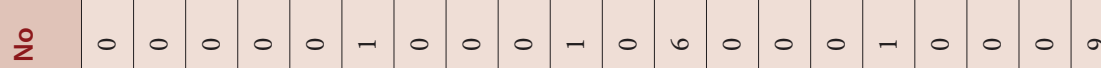

虫 


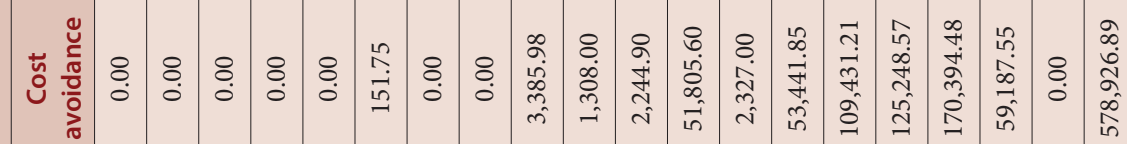

๓ัँ

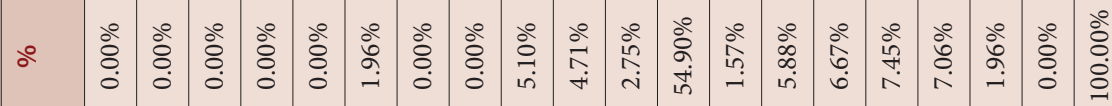

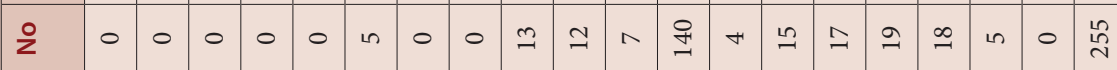

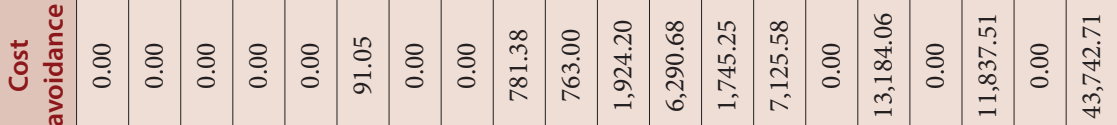

㐫

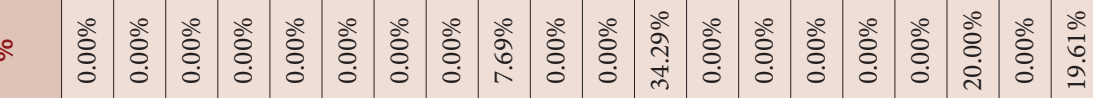

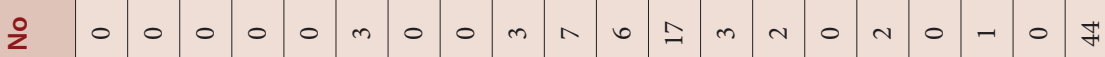

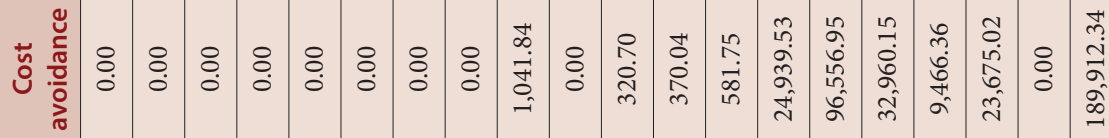

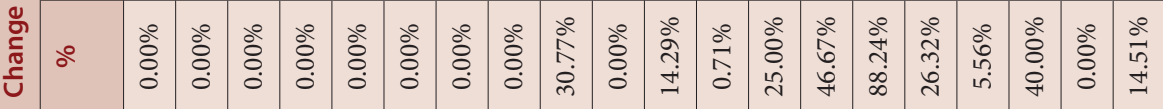

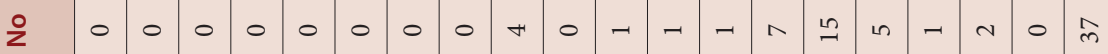

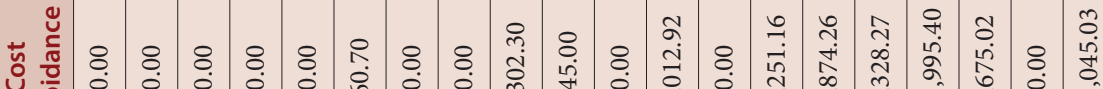

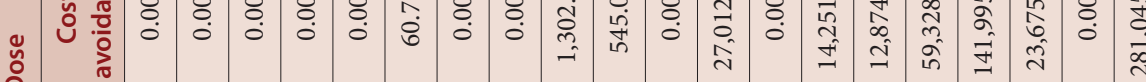

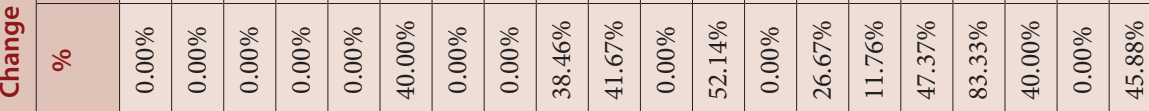

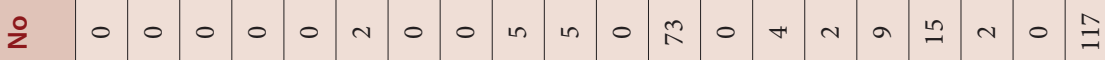

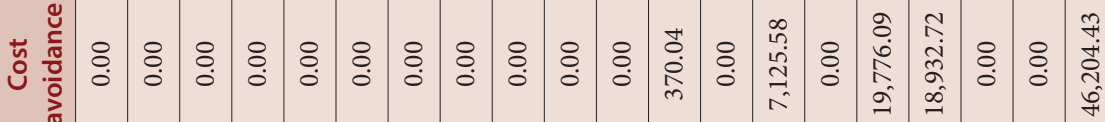

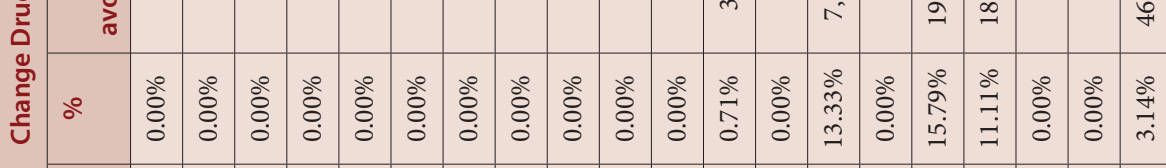

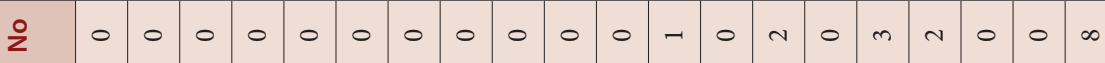

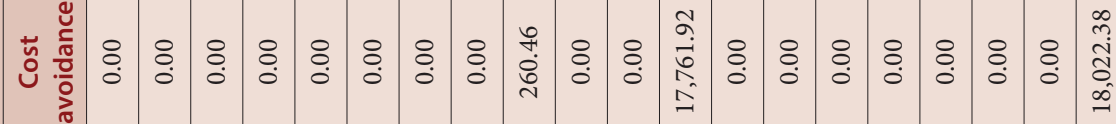

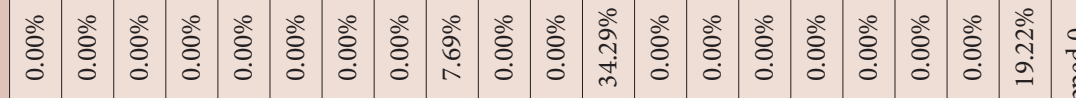

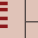

\begin{tabular}{lllllllllllllllllllllllll}
\hline & 0 & 0 & 0 & 0 & 0 & 0 & 0 & 0 & - & 0 & 0 & $\infty$ & 0 & 0 & 0 & 0 & 0 & 0 & 0 & मे
\end{tabular}

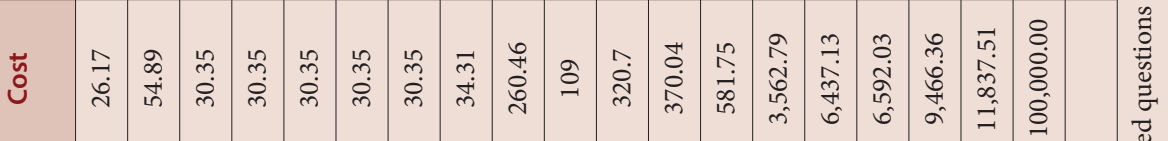

๑ 
Table 9: Patient outcome versus cost avoidance.

\begin{tabular}{|c|c|c|c|c|c|c|c|c|c|c|}
\hline \multirow{2}{*}{$\begin{array}{l}\text { Sex } \\
\text { No }\end{array}$} & \multirow[b]{2}{*}{ Cost } & \multicolumn{3}{|c|}{ Patient's Condition Improved } & \multicolumn{3}{|c|}{ Laboratory Value Improved } & \multicolumn{3}{|c|}{ Total } \\
\hline & & No & $\%$ & $\begin{array}{c}\text { Cost } \\
\text { avoidance }\end{array}$ & No & $\%$ & $\begin{array}{c}\text { Cost } \\
\text { avoidance }\end{array}$ & No & $\%$ & $\begin{array}{c}\text { Cost } \\
\text { avoidance }\end{array}$ \\
\hline $1 \mathrm{a}$ & 26.17 & 0 & $0.00 \%$ & 0.00 & 0 & $0.00 \%$ & 0.00 & 0 & $0.00 \%$ & 0.00 \\
\hline $1 b$ & 54.89 & 0 & $0.00 \%$ & 0.00 & 0 & $0.00 \%$ & 0.00 & 0 & $0.00 \%$ & 0.00 \\
\hline $2 \mathrm{a}$ & 30.35 & 0 & $0.00 \%$ & 0.00 & 0 & $0.00 \%$ & 0.00 & 0 & $0.00 \%$ & 0.00 \\
\hline $2 \mathrm{~b}$ & 30.35 & 0 & $0.00 \%$ & 0.00 & 0 & $0.00 \%$ & 0.00 & 0 & $0.00 \%$ & 0.00 \\
\hline $2 c$ & 30.35 & 0 & $0.00 \%$ & 0.00 & 0 & $0.00 \%$ & 0.00 & 0 & $0.00 \%$ & 0.00 \\
\hline $2 \mathrm{~d}$ & 30.35 & 3 & $60.00 \%$ & 91.05 & 2 & $40.00 \%$ & 60.70 & 5 & $1.96 \%$ & 151.75 \\
\hline $2 \mathrm{e}$ & 30.35 & 0 & $0.00 \%$ & 0.00 & 0 & $0.00 \%$ & 0.00 & 0 & $0.00 \%$ & 0.00 \\
\hline $2 f$ & 34.31 & 0 & $0.00 \%$ & 0.00 & 0 & $0.00 \%$ & 0.00 & 0 & $0.00 \%$ & 0.00 \\
\hline $3 a$ & 260.46 & 5 & $38.46 \%$ & $1,302.30$ & 8 & $61.54 \%$ & $2,083.68$ & 13 & $5.10 \%$ & $3,385.98$ \\
\hline $3 b$ & 109 & 4 & $33.33 \%$ & 436.00 & 8 & $66.67 \%$ & 872.00 & 12 & $4.71 \%$ & $1,308.00$ \\
\hline $3 c$ & 320.7 & 7 & $100.00 \%$ & $2,244.90$ & 0 & $0.00 \%$ & 0.00 & 7 & $2.75 \%$ & $2,244.90$ \\
\hline $4 a$ & 370.04 & 10 & $7.14 \%$ & $3,700.40$ & 130 & $92.86 \%$ & $48,105.20$ & 140 & $54.90 \%$ & $51,805.60$ \\
\hline $4 \mathrm{~b}$ & 581.75 & 3 & $75.00 \%$ & $1,745.25$ & 1 & $25.00 \%$ & 581.75 & 4 & $1.57 \%$ & $2,327.00$ \\
\hline $4 c$ & $3,562.79$ & 13 & $86.67 \%$ & $46,316.27$ & 2 & $13.33 \%$ & $7,125.58$ & 15 & $5.88 \%$ & $53,441.85$ \\
\hline $4 d$ & $6,437.13$ & 17 & $100.00 \%$ & $109,431.21$ & 0 & $0.00 \%$ & 0.00 & 17 & $6.67 \%$ & $109,431.21$ \\
\hline $5 a$ & $6,592.03$ & 18 & $94.74 \%$ & $118,656.54$ & 1 & $5.26 \%$ & $6,592.03$ & 19 & $7.45 \%$ & $125,248.57$ \\
\hline $5 b$ & $9,466.36$ & 18 & $100.00 \%$ & $170,394.48$ & 0 & $0.00 \%$ & 0.00 & 18 & $7.06 \%$ & $170,394.48$ \\
\hline $5 c$ & $11,837.51$ & 3 & $60.00 \%$ & $35,512.53$ & 2 & $40.00 \%$ & $23,675.02$ & 5 & $1.96 \%$ & $59,187.55$ \\
\hline 6 & $100,000.00$ & 0 & $0.00 \%$ & 0.00 & 0 & $0.00 \%$ & 0.00 & 0 & $0.00 \%$ & 0.00 \\
\hline Total & & 101 & $39.61 \%$ & $489,830.93$ & 154 & $60.39 \%$ & $89,095.96$ & 255 & $100.00 \%$ & $578,926.89$ \\
\hline
\end{tabular}

(325,695.76 USD) followed by incompatibility (275,802.66 USD) and inappropriate route $(189,912.34$ USD). The maximum cost avoidance in terms of pharmacist's recommendation type was recorded for change dose (281,045.03 USD) followed by change route (189,912.34 USD) (Tables 7 and 8). The maximum cost avoidance in terms of patient outcome related to was patient condition improved (489,830.93 USD) and laboratory value improved $(89,095.96)$. The maximum cost avoidance in relation to pharmacoeconomic impact was the duration of hospital stay of patient $(226 ; 332,220.70$ USD) and reduction in the cost of drug therapy monitoring (246,227.15 USD) (Tables 9 and 10).

\section{DISCUSSION}

The pharmacoeconomic program was established during pharmacy strategic planning at $\mathrm{MOH}$ in the KSA. ${ }^{1}$ Some pharmacoeconomic tools were used as the indicators to follow the implementation of pharmacy strategic plan and measure the economic outcomes of the pharmacy practice program. ${ }^{3}$ In the main public hospital in Riyadh city, the pharmacy staff implemented those tools. The authors explored the economic impact of pharmacist intervention with TPN services. The findings showed high-cost avoidance due to prevention of TPN-related problems. However, it is less than the reported value in a previous study. ${ }^{4}$ That might be because, the TPN services have been newly established, TPN pharmacist newly trained and newly started TPN services at the hospital. There is not of much a difference in the gender and related cost avoidance because most of the TPN-related problems were similar. The results showed two-third of the problems were related to Saudi patients, whereas one-third of them were related to non-Saudi patients. This is expected because most of our patients were Saudi nationals. The majority of the cost avoidance were recorded for neonates and pediatrics as most of the TPN candidates were pediatric patients. Most of the cost avoidance was recorded by senior registrar physician because of most of them committed potential mistakes of total parenteral nutrition. The maximum cost avoidance was recorded for potentially severe or significant interventions because the complications would be dangerous and highly risky to the patient even with the low number of interventions. ${ }^{13}$ The maximum cost implicated for TPN problems were general TPN consultations and TPN incompatibility; however, these problems lead to significant complications and great sequences of additional cost. Consequently, a great deal of cost is saved. The maximum cost avoidance was recorded for patient conditions improved. This might be related to the reduction of duration of hospital stay and drug therapy monitoring. That is be expected with serious or significant TPN related problems. The economic impact of TPN pharmacist was very substantial. The economic impact documentation of prevention intervention with TPN services is highly recommended at $\mathrm{MOH}$ hospitals in the KSA.

\section{CONCLUSION}

The economic outcomes of pharmacist intervention through TPN services is vital. In future, we should target to expand the TPN services managed by the pharmacist at all $\mathrm{MOH}$ healthcare institutions in the KSA.

\section{ACKNOWLEDGEMENT}

None. 


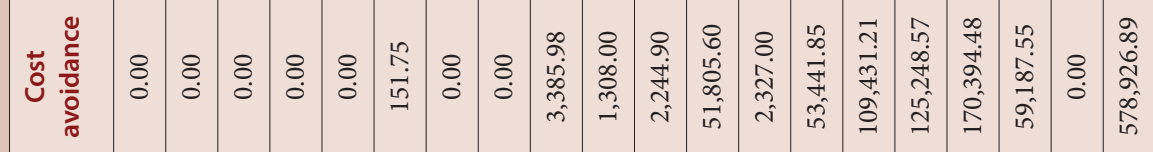

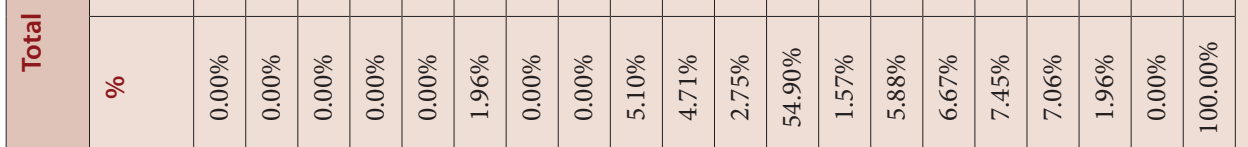

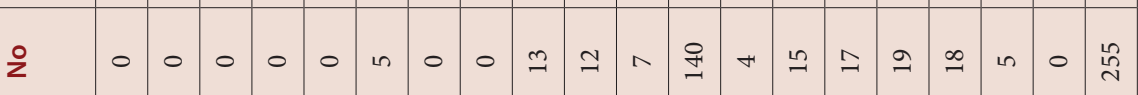

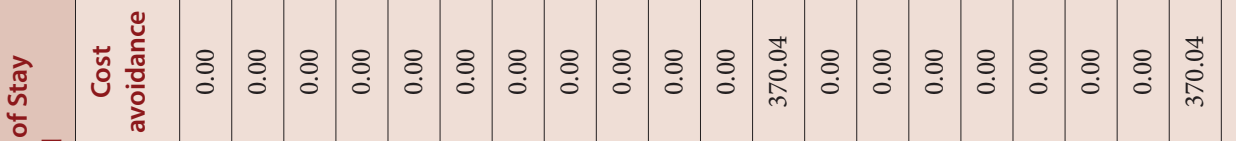

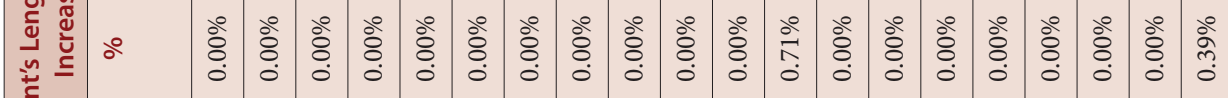
$\frac{\sqrt{2}}{\frac{0}{0}}$

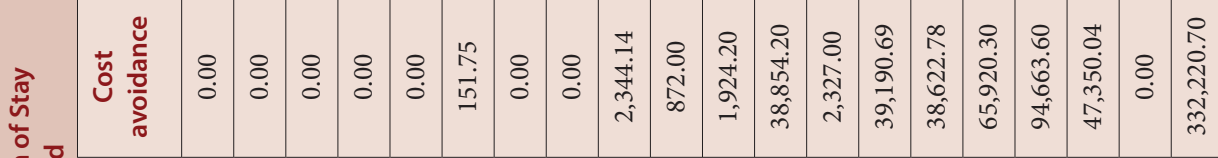

范

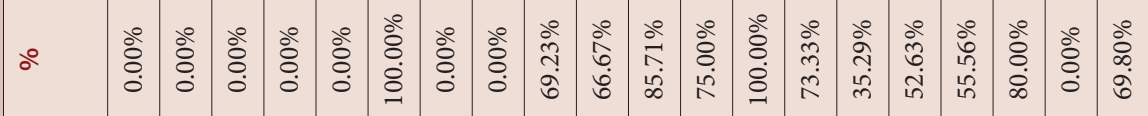

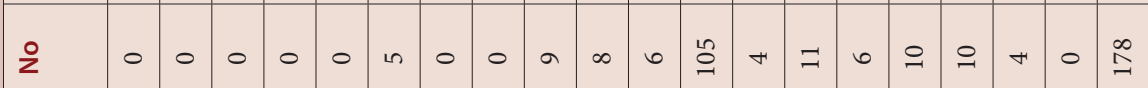

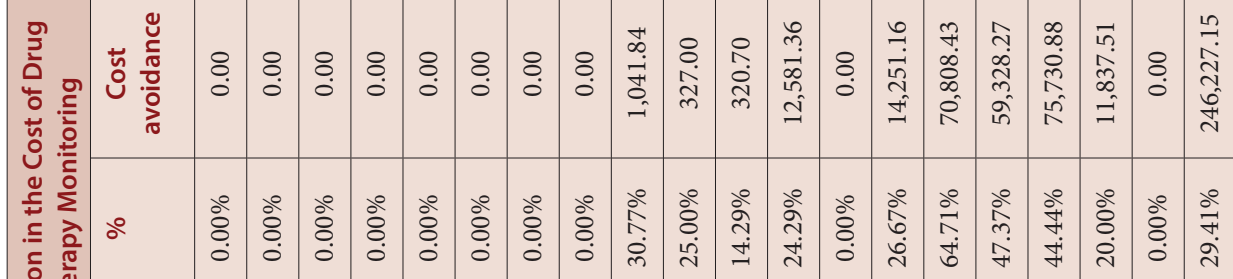
至

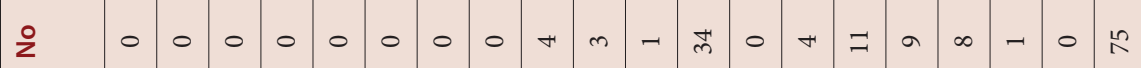

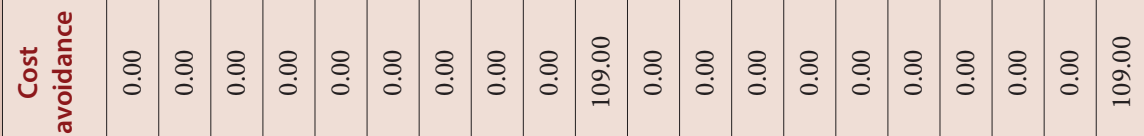

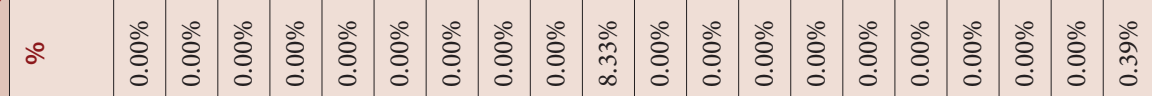
$\begin{array}{llllllllllllllllllllllllllll}2 & 0 & 0 & 0 & 0 & 0 & 0 & 0 & 0 & 0 & -1 & 0 & 0 & 0 & 0 & 0 & 0 & 0 & 0 & 0 & -1\end{array}$

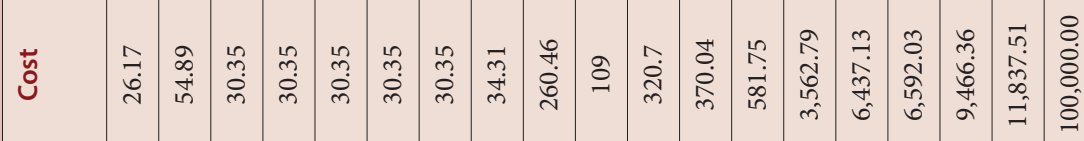

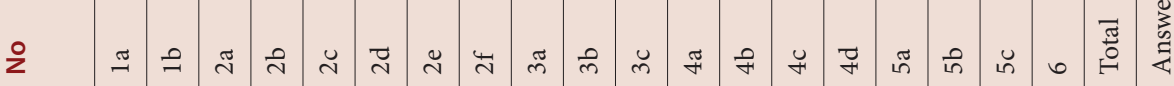




\section{CONFLICT OF INTEREST}

The authors declare that there are no conflict of interest.

\section{ABBREVIATIONS}

TPN: Total parenteral nutrition; ASPEN: American Society of Parenteral Nutrition; NICU: Neonatal intensive care unit; PICU: Pediatric intensive care unit; MOH: Ministry of Health; KSA: Kingdom of Saudi Arabia; ISMP: Institute for Safe Medication Practices.

\section{ORCID ID}

Yousef Ahmed Alomi (iD https://orcid.org/0000-0003-1381-628X

\section{REFERENCES}

1. Alomi YA, Alghamdi SJ, Alattyh RA. Strategic Plan of General Administration of Pharmaceutical Care at Ministry of Health in Saudi Arabia 2012-2022. JPharm Pharm Scien. 2015;1(13):1-8.

2. Alomi YA, Alghamdi SJ, Alattyh RA, Elshenawy RA. The Evaluation of Pharmacy Strategic Plan in Past 2013-2016 and Forecasting of New Vision 2030 at Ministry of Health in Saudi Arabia. J Pharm Pract Community Med. 2018;4(2):93-101.

3. Alomi Y. National Pharmacy Administration Programs. BAOJ Pharm Sci. 2015;1(2):1-2.

4. Bond CA, Raehl CL, Franke T. Clinical Pharmacy Services, Pharmacy Staffing and the Total Cost of Care in United States Hospitals. Pharmacotherapy. 2002;22(2):134-47

5. Katoue MG. Role of pharmacists in providing parenteral nutrition support:
Current insights and future directions. Integr Pharm Res Pract. 2018;7:125-40.

6. Meers G, Noerens K, Collier H, Cortoos P. Clinical pharmacist interventions on parenteral nutrition appropriateness in a teaching hospital. Eur J Hosp Pharm. 2015;22(Suppl 1):A1.3-2.

7. Alomi YA, Fallatah AO. Cost Avoidance Of Pharmacist Running Pediatrics Total Parenteral Nutrition Services At Ministry Of Health In Saudi Arabia. Value Heal. 2016;19(7):A461.

8. Alomi YA, Alanazi AA, Alsallouk SA, Almaznai MM, Abu-Alnaja NI, Alduhilan M, et al. Cost-efficiency of Medication Safety Program at Pediatrics, Obstetrics and Gynecology Hospital, East Province, Saudi Arabia. Value Heal. 2016;19(7):A464.

9. AlomiYA, Almudaiheem HY, Alarnous T, Alshurei S, Alsharafa A, Alzahrani T, et al. Cost-efficiency of National Drug Information Center Through Ministry of Health Hotline Calling Services (937) in Saudi Arabia: Application of American Model. Value Heal. 2015;18(7):A735.

10. Alomi YA. Cost-efficiency of Clinical Pharmacy Services at Ministry Of Health In Saudi Arabia Application of American Model. Value Heal. 2016;19(7):A482-3.

11. Ling JM, Mike LA, Rubin J, Abraham P, Howe A, Patka J, et al. Documentation of pharmacist interventions in the emergency department. Am J Heal Pharm. 2005;62(17):1793-7.

12. Mutnick AH, Sterba KJ, Peroutka JA, Sloan NE, Beltz EA, Sorenson MK. Cost savings and avoidance from clinical interventions. Am J Heal Pharm. 1997;54(4):392-6.

13. 13. Cohen MR, et al. ISMP Medication Safety Self-Assessment for High-Alert Medications. 2017. 